\title{
LA DÉMOCRATIE POPULAIRE ET L'ESTHÉTISME IRRESPONSABLE
}

Popular democracy and irResponsible aestheticism

A DEMOCRACIA POPULAR E O ESTETISMO IRRESPONSÁVEL

Crina Bud

Universidade Técnica Cluj-Napoca

Centro Universitário Nord Baia Mare

Baia Mare, Romênia

Palavras-chave: democracia, comunismo, formalismo, engajamento, responsabilidade.

Keywords: democracy, communism, formalism, commitment, responsibility.

Cuvinte cheie: democraţie, comunism, formalism, angajare, responsabilitate.

\section{Resumo}

O presente artigo sublinha a existência de significativas diferenças entre a compreensão da democracia e da autonomia literária nas culturas ocidentais e do Leste Europeu (durante o regime totalitário). No caso específico da cultura romena, a democracia costumava ser um conceito reivindicado tanto pela política oficial do partido comunista e pelos escritores que recusavam qualquer forma de instrumentalização política da literatura. As análises dão realce à tensão entre o esteticismo e o militância, formalismo e engajamento, e o drama de ter que escolher, como escrito, entre a responsabilidade moral e social. $\mathrm{O}$ contraste entre o discurso literário e o da propaganda é enfatizado por meio do uso binário do mesmo vocabulário. Entre as esquizo-palavras capazes de transmitir a tensão entre o estético e o político, nos deparamos com: o belo, a moral, democracia, tradição, formalismo, escapismo, utopia, mensagem.

\section{Abstract}

The article underlines the existence of major differences between the understanding of democracy and that of the autonomy of literature in Western and East-European cultures (during the totalitarian regime). In the particular case of Romanian culture, democracy used to be a concept claimed by both the official politics of the communist party and the writers who refused any form of political instrumentation of literature. The analyses highlights the tension between aestheticism and activism, formalism and commitment, and the drama of having to chose,

\section{Rezumat}

Articolul subliniază existența unor diferențe majore între înțelegerea democrației respectiv a autonomiei literaturii în culturile occidentale și în culturile est-europene din perioada totalitarismului. In cazul particular al culturii române, democrația era un concept revendicat atât de politicile oficiale ale partidului comunist cât și de scriitorii care refuzau orice formă de instrumentalizare politică a literaturii. Analiza pune în evidență tensiunea dintre estetism şi activism, dintre formalism și angajare, și dramatismul alegerii de către scriitori 
as a writer, between moral and social responsibility. The contrast between the literary discourse and the discourse of the propaganda is emphasized through the binary usage of the same vocabulary. Between the schizo-words able to convey the tension between aesthetic and political, we have stopped at: beauty, morals, democracy, tradition, formalism, evasionism, utopia, message.

Le livre de Vincent Kaufmann La faute à Mallarmé. L'aventure de la théorie littéraire insiste sur la dimension politique de la théorie littéraire en France et démarre par l'affirmation que Mallarmé, Baudelaire et Flaubert "sont tous coupables d'avoir choisi l'art pour l'art et l'irresponsabilité sociale".* Le séparatisme littéraire et le formalisme sont compris comme ayant quelque chose de réactif et d'élitaire et il allait de soi qu'et “il(s) n'étai(en)t pas destiné(s) à une démocratisation de la fabrication de la littérature".*

Je voudrais présenter ici une situation inverse: la démocratisation totale de la fabrication de la littérature, au sens où n'importe qui peut écrire et doit s'adresser au peuple. Cette démocratisation extrême de la fabrication de la littérature annule tout principe de liberté et donc de démocratie, car la littérature ainsi produite doit respecter une seule loi: qu'elle soit engagée, c'est-à-dire complètement soumise au politique. Dans ces conditions, le formalisme, l'esthétisme, l'autonomie de l'art deviennent de vrais principes démocratiques. Comme on le verra, la culpabilité des écrivains y est également de mise. C'est une sorte de faute tragique, quelque chose d'inévitable.

Dans ma petite histoire des acceptions du terme démocratie dans la Roumanie communiste, les mots sont caméléonesques. La Roumanie s'est engagée depuis 1947 sur la voie - indiquée par Marx et imposée par Staline - de «la vraie démocratie». À partir de 1947, l'ancienne monarchie roumaine est transformée en république populaire. Entre 1948-1956 la Roumanie a logé le Kominform et son hebdomadaire Pour une paix durable, pour une démocratie populaire. ${ }^{1}$ Le changement d'identité politique va de pair avec

\footnotetext{
${ }^{1}$ Pour plus d'information, voir François Fejtö - Histoire des démocraties popu-
}

* (KAUFMANN, Vincent. La faute à Mallarmé. L'aventure de la théorie littéraire. Paris: Seuil, 2011: 8.)

* (KAUFMANN, Vincent. La faute à Mallarmé. L'aventure de la théorie littéraire. Paris: Seuil, 2011: 100.) 
* (Constituţia Republicii Populare Române, 1948.) le changement de l'hymne national et de la constitution du pays. Le titre de l'hymne de la nouvelle république - Brisez les menottes - insiste sur l'idée de libération et de liberté. Mais quelle sorte de liberté? L'un des premiers passages de la nouvelle constitution précise: "toute association antifasciste et antidémocratique est interdite"* puis l'État s'engage à appuyer tous ceux qui travaillent. Mais les écrivains travaillent-ils? Au niveau du discours, la Roumanie a choisi les "libertés réelles" au détriment des "libertés formelles", propres aux républiques parlementaires et aux démocraties bourgeoises. C'est ainsi que commence la guerre pour la propriété des mots (la seule propriété concevable dans ce type de démocratie à rebours). Elle atteint son comble dans les années 60-70 du communisme roumain. C'est alors que le pays est devenu une république socialiste mais la prétention de démocratie y persiste, même si le qualificatif populaire est remplacé ou seulement renforcé par d'autres adjectifs: directe, participative, plébiscitaire. C'est maintenant que la tension entre, d'une part, l'esthétisme - comme option pour l'art pur, soustrait à tout conditionnement social - et, d'autre part, l'activisme - comme instrumentalisation de l'art dans le processus de la construction du socialisme, bat son plein. L'exigence de pureté esthétique est formulée contre l'usage social de la littérature, mais ainsi son sens principal est détourné et maculé. La relation de la littérature, qui se veut autonome, avec l'idéologie communiste est analogue au regard de Nietzsche dans l'abîme ("si tu plonges longtemps ton regard dans l'abîme, l'abîme te regarde aussi"): en se pensant et s'écrivant à l'encontre du système totalitaire, cette littérature s'imprègne de quelque chose qui appartient à l'abîme politique. À partir du moment où ce piège de l'imposture est appréhendé par les écrivains puristes, une série d'effets deviennent visibles: des effets esthétiques - radicalisation et donc accessibilité auprès d'un public extrêmement restreint, en plus d'un inévitable anachronisme; des effets psychologiques - tourments sur la moralité d'éviter une confrontation directe ; et des effets comportementaux - on assume une condition d'écrivain damné, qui pourrait paraitre désuète, est assumée.

L'une des illustrations les plus percutantes de l'inconfort moral qui accompagne l'option en faveur des valeurs esthétiques dans les

laires, Seuil; 1952, Histoires des démocraties populaires après Staline, Seuil, Paris, 1969; Comisia prezidențială pentru analiza dictaturii comuniste - Raport final, Humanitas, 2007. 
années 60-70 est le cas de conscience ouvert par Ion Negoițescu et Matei Călinescu concernant la reconnaissance et en même temps l'accusation d'une faute d'esthétisme irresponsable. Une fois coincés entre les murs totalitaires, les deux critiques roumains choisissent de s'exiler. (en 1973, Matei Călinescu s'établit aux Etats Unis et devient professeur de la littérature comparée à Indiana University, célèbre pour ses livres Five faces of modernity, Rereading etc; Ion Negoițescu s'établit en Allemagne, en 1978, où il écrit une Histoire de la littérature roumaine). Aussi longtemps qu'ils sont restés à l'intérieur du système, leur option radicale pour l'esthétisme avait dans leur propre conscience la valeur d'un acte de résistance contre le siège idéologique. Mais scruté du dehors, avec la distance qu'offre l'exil, cet exclusivisme perd son aura héroïque et tourne en une culpabilité exprimée avec intransigeance par les deux critiques.

Refuser à la littérature toute fonction sociale, dans les conditions où le régime politique était prêt à la lui reconnaître et se servir de cette unique dimension, c'était une déclaration d'indépendance et une affirmation d'identité. En 1987, Ion Negoițescu reproche aux écrivains roumains et à lui-même l'esthétisme irresponsable, c'est-àdire "le manque de réaction politique contre le mal qui faisait que la Roumanie en tant qu'entité historique créatrice s'anéantisse».* En 1991, après la chute du communisme et des murs qui en protégeaient le mystère tout en l'accroissant, Matei Călinescu adhère au jugement intransigeant de son ami Ion Negoițescu et ajoute de nouvelles rubriques à la symptomatologie de ce mal de l'histoire nommé esthétisme irresponsable. Si le choix de l'autonomie de l'art n'est jugé qu'une preuve de professionnalisme ("la morale des artistes en tant qu'artistes"), le consentement à "la lobotomie politique, c'est-à-dire la sévère atrophie du sens politique", ${ }^{*}$ est perçu comme une amputation morale, comme un professionnalisme irresponsable.

Virgil Ierunca, l'une des voix roumaines les plus importantes de la radio Europe Libre a publié une Anthologie de la honte comprenant des textes de démission morale des intellectuels roumains face aux sollicitations politiques. Mais voilà qu'à travers les questions que se posent Negoițescu et Călinescu, quant à une présumée faute esthétique, un autre type de honte surgit, qui est plus subtile, mais d'autant plus authentique et plus problématique: la honte d'avoir pris la littérature pour un refuge, pour un espace de braconnage esthétique dans les champs minés et sévèrement sur-
* (NEGOITESCU, lon. În cunoştinţă de cauză. Dacia: Cluj Napoca, 1990: 85.)

* (CALINESCU, Matei. Estetismul nostru iresponsabil. Meridian: Juillet-Aout, 1991: 57.) 
* (VIANU, Lidia. Cicatricea intelectuală. Interview avec Matei Călinescu, România literară, 4-10 Mars 1993: 13.) veillés de l'idéologie, la honte de s'être laissé aller à l'illusion que l'esthétisme est un acte de courage et puis l'immense désillusion de cette prise de conscience puisque ce courage restait dans les limites convenues par la censure. L'effet que Matei Călinescu appelait post-censure est un exemple éloquent du piège psychologique dans lequel se sont vus pris ces adeptes de la littérature comme littérature: après la satisfaction d'avoir publié un livre et donc après une victoire sur la censure naît "un sentiment de vanité, de honte, d'épuisement"; du moment que le texte a été publié, c'est qu'il représentait l'épreuve d'une négociation entre l'esthétique et le politique. Le résultat est "une cicatrice intellectuelle", ${ }^{*}$ un stigmate intérieur assez difficile à porter.

Dans ces conditions favorisant toutes les cohabitations paradoxales, l'idée de l'autonomie littéraire n'a rien de la gratuité ou de l'amoralité de l'art pour l'art, tel que ce concept était utilisé au XIXe siècle. L'autosuffisance de l'art pour l'art exclut l'idée de justification de soi car l'art est son propre but. Mais le contexte historique dont on parle rend impossible un tel espace aseptique, dépourvu de connexions, pour la littérature. L'époque staliniste des prisons, de l'élimination physique ou seulement intellectuelle (par la stricte interdiction de publier) est révolue. De même, les règles sévères du réalisme socialiste, comme unique manière de création, sont relâchées, et le slalom entre les slogans de la propagande devient possible. Le socialisme se donnait la peine de poser, y compris aux yeux de ses propres citoyens, et pas seulement à ceux de l'Occident, avec un visage humain et par conséquent démocratique. Aussi, l'action se voit-elle doubler d'une motivation discursive. Les mécanismes de la démocratie populaire et le principe totalitaire qui l'appuie imposent cet ordre à l'envers: agir tout d'abord et ensuite légiférer l'action, ordre qui offenserait n'importe quelle utilisation naturelle du mot démocratie. Les textes officiels de l'époque abordent de plus en plus fréquemment l'idée de la politique culturelle de la Roumanie, avec une gravité feinte, doublée d'un cynisme trahi par l'affirmation du général de la Sécuritate Nicolae Pleșiţa: "il fallait verser de la culture dans l'appareil” (Ochii și urechile poporului, 2001).

La machine totalitaire avait besoin du lubrifiant culture et c'est la raison pour laquelle, par une étrange appropriation de compétences, Nicolae Ceaușescu devient critique littéraire, ainsi qu’il sera ironiquement désigné plus tard. Dès 1965, il fait entrer dans les tables de lois du nouveau monde trois interdictions qui visent 
sans aucune trace de confusion la subordination de la littérature au politique et donc la mise en illégalité de la prétention d'autonomie du domaine littéraire: le formalisme, le langage diffcile et les thèmes inacceptables (la solitude, l'aliénation, la mort). La présence de Ceaușescu parmi les écrivains se voulait souvent accueillie par des déclarations-engagement, comme il est arrivé en 1968, lorsqu'une commission de l'Union des écrivains était tenue de préciser: "Le marxisme rejette fermement la conception d'une soi-disant indépendance ou bien de l'autonomie de l'art vis-à-vis de la société".* Cette époque était encore celle de la popularité, on mimait le dialogue arbitré par la voix suprême de Marx. On mettait l'accent sur la fonction formative de l'art exprimée par Marx et renversée par les exécutants-activistes dans sa réalité grotesque. Mais à partir de 1971, on abandonne la fonction éducative de l'art en faveur d'une attitude guerrière: l'art n'est pas médiateur, il doit conquérir. C'est Ceaușescu lui-même qui annonce cette radicalisation lors d'une célèbre rencontre avec les écrivains à Neptun, au bord de la Mer Noire. Contrarié d'avoir été obligé de renoncer à un match de volleyball pour résoudre les problèmes de "ces auteurs des Roméos et Juliettes", le président, sous l'ombrelle du nationalisme, prononce le verdict: "la liberté de création ne signifie pas du tout la liberté d'embrasser des idéologies étrangères à notre régime socialiste. La littérature roumaine doit être une littérature socialiste militante."*

Dès que Ceaușescu a déclenché sa mini-révolution culturelle, on redouble les efforts de justification interne et externe.

Le soin pour l'image de la culture roumaine à l'étranger se manifestait déjà dans les années 60 lorsqu'on a fondé un Institut pour les relations culturelles avec l'étranger. La mission de l'Institut était d'inviter en Roumanie des intellectuels de l'étranger et de les convaincre des avantages "d'une démocratie de style latin". Parmi les invités il y avait aussi des Français. Quelques-uns d'entre eux ont contribué au numéro spécial de la revue Europe de 1959, numéro dédié à la littérature roumaine. Au nom de la démocratie, Pierre Abraham et Pierre Paraf s'enthousiasment devant le palais de Sinaia qui avait appartenu aux anciens rois de Roumanie, mais qui à ce moment-là était devenu «la maison de repos des syndicats et maison de méditation et de travail pour les écrivains". * Pendant que l'on montrait aux visiteurs occidentaux les grandioses réalisations de la Roumanie communiste, pendant qu'une partie de ces visiteurs vantaient les extraordinaires conditions offertes par le
* (GABANYI, Annely Ute. Literatură şi politică în România după 1945. Bucureşti: Editura Fundaţiei Culturale Române, 2001: 175.)

* (MALITA, Liviu. Ceauşescu critic literar. Bucureşti: Vremea, 2007: 52.)

* (Europe, 37 Année, 363364, 1959: 9.) 
* (BALAN, lon Dodu. La politique culturelle en Roumanie. Paris: Les Presses de I'UNESCO, 1974: 18.)

* (BALAN, Ion Dodu. La politique culturelle en Roumanie. Paris: Les Presses de I'UNESCO, 1974: 20.) régime aux "serviteurs de l'Esprit", les intellectuels les plus importants de Roumanie moisissaient dans les prisons politiques (y compris Negoițescu). Mais cela va de soi, les fonctionnaires culturels roumains évitaient de parler de ces facettes de la démocratie populaire. Les autres anthologies de littérature roumaine publiées en français, respectivement en 1962 (Nouvelles roumaines. Anthologie des prosateurs roumains, Seghers) et en 1968 (Anthologie de la poésie roumaine, Seuil), faussaient l'image de la littérature roumaine, et les critiques roumains (Tudor Vianu et Serban Cioculescu) qui cautionnaient la sélection et la présentation critique abdiquaient en égale mesure aussi bien de la responsabilité esthétique que de la responsabilité sociale.

Dans les années 70 , on publie des compendiums, des comptes rendus, des anthologies de textes signés aussi par des auteurs qui, de par leur qualité morale ou artistique, pouvaient cautionner le nouveau virage politique au prix de se voir entacher leur réputation. On publie, en français, le volume collectif La Roumanie et sa politique culturelle (1973), en roumain, l'anthologie La culture socialiste en Roumanie, en français, ainsi qu'un texte de Ion Dodu Blan rédigé à la demande de UNESCO: La Politique culturelle en Roumanie (1974). Le volume UNESCO débute cyniquement par la mise en évidence de la contribution de Roumains comme Eugène Ionesco, Emile Cioran, Georges Enescu ou Brancusi (artistes indésirables en Roumanie à l'époque) à la culture européenne. Son contenu peut être réduit à une inclusion: "la culture n'est plus l'apanage d'une élite mais le bien des masses" et à deux exclusions: "ni l'idéalisme, ni le nihilisme ne sont compatibles avec l'essence des arts réalistes" et "il n'y a pas de place pour une création sans finalité sociale".* On est surpris par l'enthousiasme en ébullition de la phrase synthétisant le programme communiste et visant un sentiment esthétique: "en remplaçant la routine et les préjugés de quelques fonctionnaires par l'initiative créatrice de masses éprises de beauté".*

Qu'il est étrange ce pan-esthétisme communiste clamé contre l'art autonome! On dirait que les mots n'ont jamais été plus dépourvus d'une substance propre, plus impuissants devant les tentatives de prise en possession par un langage artificiel, parfaitement instrumentalisé. On pourrait établir un jour un dictionnaire des schizomots, des mots "fendus" qui pourraient fonctionner à la fois dans le champ esthétique et politique. Y entreraient les mots aux sens détournés par la propagande communistes, des mots lavés idéolo- 
giquement pour qu'ils servent ensuite, par répétition automatique, au lavage des cerveaux, beaucoup plus grave, auquel s'adonnait le programme totalitaire. Le mot beauté, mentionné ci-dessus, ferait partie de ce vocabulaire clivé. Si Walter Benjamin dénonce l'esthétisation fasciste de la politique, un processus similaire peut être identifié dans le cas du communisme. Depuis la fabrication marxiste de l'histoire par ce que Boris Groys appelle l'œuvre d'art totale de Staline et par l'esthétisation du monde ciblé par les surréalistes, la transfiguration du réel par l'esthétique atteint un comble roumain dans l'image électrisée des masses éprises de beauté. (Il est à préciser que chez Marx l'esthétique n'était qu'un véhicule qui servait à fabriquer l'histoire, tandis que la démocratie n'était qu'une simple étape transitoire vers la dictature prolétaire et ensuite vers la disparition de l'Etat et la sortie de l'histoire.)

Pendant que la démocratie populaire incite l'appétit créateur de masses, les adeptes de l'esthétisme, des individualistes convaincus, ne cessent de croire à "une mystique de la littérature" (Negoițescu) et à définir la dimension esthétique d'une œuvre comme "une cohérence intérieure, secrètement poétique, d'une personnalité qui s'est dédiée à l'exercice tellement difficile et dangereux de la lucidité".* Après trois ans de prison politique et d'interdiction à publier, Ion Negoițescu revient dans la littérature roumaine avec un désir impétueux d'identifier les sèves, les ferments esthétiques des œuvres, avec un effort de recherche du "lyrisme phréatique". En 1968, il publie dans la revue la Famille* le schéma d'une histoire de la littérature roumaine qu'il projette comme un effort d'écorcer la littérature roumaine de toutes ses couches extra-littéraires pour en venir à son noyau esthétique, le seul qui justifie sa contribution aux valeurs européennes. La réaction des autorités est véhémente, les critiques au service du pouvoir s'attaquent de manière hystérique à un tel projet, d'autant plus que la même année on avait publié (pour être immédiatement interdite) une Anthologie de la poésie moderne réalisée par N. Manolescu. Les deux auteurs sont accusés d'illégalité critique et le plaidoyer final des adversaires soutient qu' 'au nom de l'autonomie des valeurs esthétiques du passé, ils rejettent avec véhémence des œuvres qui expriment des points de vue démocratiques"* (c'est-à-dire les œuvres qui évoquaient des tracteurs, des fourneaux, des sondes, des usines, le plan quinquennal, l'émulation dans le travail, l'amour sous les étendards rouges du communisme, etc). De l'autre côté, après un volume flanqué
*(CALINESCU, Matei. Eseuri critice. Bucureşti: EPL, 1969: 11.)

* (NEGOITESCU, lon. Istoria literaturii române-planul. Familia, 2, 1968:7.)

*(GABANYI, Annely Ute. Literatură şi politică în România după 1945. Bucureşti: Editura Fundaţiei Culturale Române, 2001: 168.) 
*(CALINESCU, Matei. Fragmentarium. Dacia, 1973: 45.) de concessions tactiques et de rubans de conformisme idéologique, le plus jeune Matei Călinescu vire définitivement vers une définition strictement esthétique de la littérature et comprend l'acte critique comme la tentative de constituer un concept de la littérature et comme un essai de découvrir la justification du sens de la tradition. ${ }^{*}$

Et voilà bien un autre noyau de conflit : la tradition, qui ferait elle aussi bonne figure dans notre schizo-dictionnaire. L'art engagé doit effacer les valeurs esthétiques du passé en cochant l'un des articles du programme plus large exprimé par les vers de l'Internationale "du passé faisons table rase", tandis que les esthètes s'efforcent de légitimer une tradition. D'où ce positionnement antithétique face au sens de l'histoire: les activistes en avant-garde, les esthètes en arrière-garde, les uns en permanente offensive, les autres en défendant leur droit à l'autonomie et à la mémoire culturelle. La génération 60 , née après la sécheresse dévastatrice du réalisme socialiste, n'écrit pas au rythme de sa contemporanéité mais avec les instruments de la modernité, avec les moyens de ses précurseurs de l'entre-deux-guerres. Ce retard est le prix à payer pour l'autonomie de la littérature et une passerelle jetée sur l'abîme proletcultiste afin de relier avec la tradition, mais aussi un pas en arrière, pour sortir de la portée des flèches idéologiques...

L'épreuve de l'anachronisme, de l'isolement et finalement de l'auto-condamnation au silence est celle de la confrontation aux standards occidentaux (eux-mêmes basés sur le conflit entre l'art autonome et l'engagement). En tant que boursier, Negoițescu quitte la Roumanie en s'engageant à écrire une étude sur Dostoïevski. De Munich, il envoie à ses amis Virgil Nemoianu et Matei Călinescu - universitaires aux Etats Unis - des fragments à publier. Malgré la reconnaissance de ses idées toujours originales et de son expression baroque surprenante, Virgil Nemoianu ne saurait ignorer la totale étrangeté et inadéquation de son style au modèle américain. Cet écartement stylistique est dû à un exercice prolongé de l'expression ésopique du message caché entre les mots et les lignes. Matei Călinescu avait senti, lui aussi, le goût amer de l'intraductibilité culturelle quand il avait essayé de publier la traduction en français de son roman La vie et les opinions de Zacharias Lichter chez une maison d'édition de Lausanne. Les confessions de l'auteur à propos de son impuissance et de son refus de relire son propre roman après avoir quitté la Roumanie sont particulièrement intéressantes. En Roumanie son livre a été interprété comme une forme de résis- 
tance au communisme, mais au lieu d'en ressentir une quelconque satisfaction, l'auteur éprouve un doute aigu quant à la qualité esthétique de son œuvre. Ce n'est là qu'une des nombreuses situations où l'on peut observer que, comme à l'intérieur d'une clepsydre, ce qu'on gagne du côté du message subversif diminue d'autant du côté de l'esthétique et vice-versa (quand la responsabilité esthétique se manifeste, la responsabilité sociale s'efface). Des livres prisonniers de l'histoire, des livres sous l'exécration de la peau de chagrin, sources de chagrin.

L'accusation la plus fréquente à l'adresse des esthètes vise leur formalisme jugé d'être excessif. Animés un certain temps par des idéaux convergents à ceux des bolchéviques, les formalistes russes en arrivent à séparer la littérature de la société et cette attitude va les chasser du paradis en train d'être bâti. Le formalisme reproché aux écrivains roumains était par conséquent une sorte de péché originel lié aux manières de comprendre et d'envisager la littérature. Dans la stricte contemporanéité de la dispute, l'accusation de formalisme était un autre rideau de mots entre l'activité littéraire roumaine et les révoltes du structuralisme et de la théorie littéraire en général. L'essentialisme, la subjectivité, l'œuvre en soi, l'attention accordée à la musique, l'anhistoricité - voilà autant d'éléments qui définissent, du point de vue occidental, la critique formaliste. Mais ce sont toujours eux qui donnent le droit à William Marx de parler d'un suicide de la littérature et notamment d'avancer une analogie du moins surprenante: Valéry, le principal promoteur de la critique formaliste que Negoițescu adore, "n'est rien d'autre que le Karl Marx de la littérature et la poésie pure son communisme, grâce auquel l'histoire, enfin, est dépassée".* Si William Marx a raison, alors l'évasionisme, l'autre reproche majeur fait aux esthètes, pourrait avoir une plus profonde signification dont les prosélytes marxistes roumains pourraient se douter. Car il ne s'agirait plus seulement de l'évasion de certaines personnes, à l'identité précise, loin des contraintes de la recettes de création réaliste, mais aussi de l'évasion de la littérature elle-même de ses définitions antérieures et de l'histoire (voir l'extra-esthétique ciblée par Negoițescu ou bien l'opinion de Călinescu que le message d'une œuvre a tendance à dépasser l'esthétique). Un tel saut au-delà de l'histoire, visé par les deux directions mais suivant des voies divergentes, conduit à une conclusion de parcours où les paradoxes défilent de nouveau: esthé-
* (MARX, William. Naissance de la critique moderne. La littérature selon Eliot et Valéry 1889-1945. Arras: Artois Presses Université, 2002: 127.) 
* (“Dosar de urmărire" [Dossier de surveillance] 66534, vol. 3, of CNSAS (Conseil national pour l'étude des archives de la Securitate).) tisme contre marxisme, exclusivisme esthétique contre travail, utopie contre utopie.

Une certaine irresponsabilité comportementale s'avère importante en ce qui concerne la liberté d'expression. Les officiers de la Sécuritate et les mouchards pouvaient par exemple pardonner à Ion Negoițescu ses extravagances et ses opinions tranchantes parce qu'il était timide, naïf, homosexuel et alcoolique ou dans un autre portrait chaud-lapin et ivrogne.* A son tour, Matei Călinescu accepte l'idée qu'une certaine liberté pouvait être gagnée par une vie bohème, voire par l'alcoolisme, bien que les deux solutions se fussent avérées tragiques pour beaucoup de représentants de cette génération-là. (Pour confirmer un tel phénomène dramatique parmi les écrivains sous la pression du communisme, voir aussi l'étude de Cécile Vaissié Les Ingénieurs des âmes en chefs. Littérature et politique en URSS 1944-1989, Paris, Belin, 2008). Les trois tentatives de suicide de Ion Negoițescu (la première en 1961 dans la prison de Malmaison, la deuxième le 23 août 1974 lorsqu'on célébrait la soi-disant libération de la Roumanie par les troupes soviétiques et la troisième après son dernier arrêt, qui a eu lieu en 1977, suite à son adhésion au mouvement anti-communiste tchèque Charte 77) excluent cette perspective de la zone de l'anecdote ou du pittoresque. Ces tentatives sont les conséquences d'un désespoir strictement personnel, mais leur but était de défier un régime de l'optimisme obligatoire, dans le vocabulaire duquel le mot mort ne pouvait même pas figurer (voilà donc un terme que l'histoire n'a pas pu scinder et pour cela il se soustrait au schizo-dictionnaire lui-même).

Par rapport à qui ou à quoi ces écrivains étaient-ils irresponsables? Quand est-ce que Ion Negoițescu a été irresponsable? Lorsque, au nom de valeurs esthétiques, il essayait de légitimer une nouvelle direction au sein de la littérature roumaine, ayant comme enjeu la littérature d'idées de jeunes écrivains comme Toma Pavel, Matei Călinescu, Virgil Nemoianu, ce qui lui a valu que tout le tirage du livre La lampe d'Aladin (1971) soit anéanti? Lorsque, en 1977 , il fut le seul écrivain roumain qui ait signé la lettre d'adhésion au mouvement anti-communiste tchèque la Charte 77 , geste qui lui a valu une nouvelle arrestation? Ou bien quand - cédant à la pression de l'enquête, il écrit un article pour se rétracter?

Etaient-ils irresponsables, tous les critiques roumains militant pour l'autonomie de l'art qui envisageaient la littérature comme une forteresse au sein de laquelle les valeurs culturelles et humaines 
pouvaient survivre? Leur faute était de pratiquer tout simplement la littérature comme littérature, mais dans le contexte donné, leur entêtement d'être écrivains et rien d'autre courait le risque d'être l'équivalent d'un Fiat ars pereat mundus. Ion Negoițescu décrit la Roumanie des dernières décennies du nationalisme-communisme comme "une chambre dans laquelle gisait un mort enveloppé dans le drapeau tricolore». L'isolement et l'aliénation extrême de la Roumanie apparaissent plus choquants encore si l'on fait une comparaison avec d'autres pays du bloc communiste (Tchécoslovaquie, Pologne, Hongrie) où les voix des écrivains étaient plus sonores dans l'espace public et où leurs prises de position contribuaient à rapprocher leurs sociétés de l'idée de démocratie.

Toutes les questions sur la responsabilité cherchent des réponses qui ne peuvent pas être formulées en l'absence d'un repère fondamental: la liberté. Georges Konrad, dans la préface du célèbre livre de Miklos Harastaszi La prison de velours, décrit l'absurde utilisation du concept de responsabilité au sein de l'Etat totalitaire: "Dans l'Etat socialiste on ne parle jamais de la liberté des écrivains, on parle toujours de la responsabilité des écrivains. Celui qui parle de la liberté est irresponsable.”* Par leur option pour l'esthétisme, une partie des écrivains roumains parlaient en fait de la liberté, même s'ils le faisaient d'une manière sophistiquée où à voix basse. La liberté obtenue par l'exil ou par la chute du communisme rend possible la distinction entre responsabilité artistique et responsabilité sociale. A partir de là, tout un processus moral a commencé, lequel crée encore des tensions au sein de la culture roumaine. De nombreux critiques et écrivains roumains jugent la culpabilité ou l'héroïsme de la "résistance par culture" et leurs voix exigeantes font recours à des mots délivrés de la captivité de sévères dictionnaires idéologiques ou esthétiques. Délivrés d'adjectifs-menottes - message politique/message littéraire, réalisme socialiste/réalisme essentiel, libertés formelles/libertés réelles, amoralisme esthétique/ amoralisme impérialiste, ces mots libres (la démocratie sans adjectifs)* rendent légitimes les questions morales.

Crina Bud é PhD Associate Professor na UniversidadeTécnica Nacional de Cluj Napoca. Publicou três livros e diversos artigos. Dentre seus temas de trabalho encontram-se a literatura e o totalitarismo, a conexão entre a história literária e a história cultural, e as configuraçóes críticas da literatura romena no exterior. E-mail: <crinabud2003@yahoo.fr>
* (HARASZTI, Miklos. The Velvet Prison - Artists under State Socialism, translated by K. Landesmann and S. Landesmann. Penguin, 1989: xiv.)

* (Dictionnaire du communisme, sous la direction de Stéphan Courtois, Larousse, 2007: 186.)

Recebido em 20/07/2013

Aprovado em 10/09/2013 